\title{
What is the Evidence for Dark Matter?
}

\author{
J. A. Sellwood \\ Department of Physics \& Astronomy, Rutgers University, \\ 136 Frelinghuysen Road, Piscataway, NJ 08855
}

\begin{abstract}
Newtonian mechanics indicates that galaxies and galaxy clusters are much more massive than we would have guessed from their luminosities, with the discrepancy being generally attributed to dark matter halos. An alternative hypothesis is that accelerations in very weak gravitational fields are larger than predicted by Newton's laws, and there is no need for dark matter. Even though we do not currently have a satisfactory theory associated with this rival hypothesis, we can ask whether any observational tests could rule it out or prefer it over the dark matter hypothesis. Current evidence suggests that neither hypothesis enjoys a decisive advantage over the other. If dark matter turns out to be the correct interpretation however, then theories of galaxy formation face some quite severe fine-tuning problems.
\end{abstract}

\section{Introduction}

The success of the currently favoured $\Lambda$ CDM model for structure formation (e.g. Bahcall et al. 1999; White, this meeting) has persuaded many people to abandon alternatives (e.g. Binney, these proceedings). But the absence of a well-worked alternative should never be sufficient reason to decide that a particular model is correct. Despite dark matter being a central ingredient of this popular model, our only evidence for its existence is the gravitationally inferred mass discrepancies, and it is becoming clear that there are significant difficulties with its apparent properties (e.g. Ostriker \& Steinhardt 2003). Furthermore, alternative gravity ideas continue to meet with some success. I therefore prefer to keep an open mind until experimental evidence for one or other interpretation of mass discrepancies becomes compelling.

Here I review the rationale for alternative gravity theories (§2) and argue (§3) that galaxy formation theory does not provide decisive evidence in favour of dark matter. I then try to outline possible experimental ways in which the two hypotheses to account for mass discrepancies could be distinguished. If dark matter is an elementary particle, then it may be possible to detect it directly (see $§ 4$ ). Alternatively, two types of astronomical observations might favour one or other interpretation: (a) the acoustic oscillations in the cosmic microwave background ( $(5)$, and (b) whether light is a good tracer of the mass. The second is a many faceted question, ranging from the issue of whether the rotation curve of a galaxy can be predicted from its luminosity profile $(\S 6)$, to whether the dark matter halos have a different shape, or are misaligned with the light, or have 
substructure not associated with luminous features ( $\S 7)$. Finally, I review some theoretical arguments related to mergers $(\S 8)$.

\section{Modifications to basic laws}

The puzzles presented by dark matter and especially by dark energy are severe, but not yet sufficient to compel us to reject the laws that seem to require their existence. Yet it is legitimate to explore whether alternative laws could lead to a simpler and more beautiful picture. A simplified picture would be attractive only if it were consistent with all current data, and compelling if it could make testable predictions that differ from those of the standard paradigm. Unfortunately, no suggestion as yet comes close to meeting these ideals.

The apparent flatness of the universe (e.g. Hinshaw et al. 2003) is generally interpreted to imply a critical energy density in a Friedmann model. The current $\Lambda$ CDM model supposes the energy density to be composed of an ugly mixture of $\sim 70 \%$ dark energy, $\sim 25 \%$ dark matter, $\sim 4 \%$ baryonic matter, and maybe as much as $1-2 \%$ in neutrinos, although this last number is an upper limit that may well be much less. Not only is it unattractive to have several components having similar densities, but the interpretation of the dark energy as Einstein's cosmological constant requires us to live at a special time just when the matter and dark energy densities are comparable; matter was dominant until relatively recently, and dark energy is now becoming dominant as the expansion reaccelerates. Finally, the value of the vacuum energy density is lower than naïve estimates based on elementary quantum mode counting by some 120 orders of magnitude, a discrepancy for which physics is completely unable to account (e.g. Weinberg 2000). Tracking quintessence models (e.g. Wang et al. 2000) and cyclic universe models (e.g. Khoury et al. 2003) answer some of these objections, while Padmanabhan \& Choudhury (2002) suggest that perhaps a single scalar field could account for both the dark energy and the dark matter an idea that would seem to merit further investigation.

The particularly severe problems presented by dark energy have driven even mainstream physicists (e.g. Arkani-Hamed et al. 2002; Carroll et al. 2003) to speculate that it may instead reflect a breakdown of general relativity on the horizon scale. But there are far fewer physicists who would argue that dark matter, for which the evidence is also exclusively gravitational, is another manifestation of new gravitational physics. One reason for this different attitude is that a weakly-interacting, massive particle (WIMP), of the kind predicted by supersymmetry, would survive as a relic from the early universe with an expected abundance of order the critical density (e.g. Kolb \& Turner 1990, §5.6).

It has therefore been left to a rather renegade group of mostly astronomers to argue for alternatives to dark matter, and several such ideas have been reviewed at this meeting by Aguirre (these proceedings). By far the best known of these alternative ideas is MOND, first proposed by Milgrom (1983), with a more sophisticated formulation being given by Bekenstein \& Milgrom (1984). Their original proposal was a modification of Newton's law of gravity that would automatically ensure asymptotically flat rotation curves for galaxies and the TullyFisher law of the form $M \propto V_{\text {flat }}^{4}$, with no requirement for dark matter. This proposal has led to a fitting formula for galaxy rotation curves of remarkable 
power (see below), but as a theory of gravity (or of inertia) it is very poorly founded, probably wrong in details (at least), makes no clear predictions about the gravitational deflection of light, and does not remove the need for all dark matter in galaxy clusters (Aguirre, Schaye \& Quataert 2001; Sanders 2003).

Partly for these reasons, I do not focus on MOND, but attemp't to enumerate possible tests that could distinguish between dark matter and any generic alternative gravity hypothesis.

\section{Structure and galaxy formation}

The hierarchical clustering of galaxies is believed to have arisen from almost homogeneous initial conditions through gravitational instability. It is well-known that Newtonian gravity arising from baryonic perturbations alone could not drive the evolution from the observed tiny fluctuations in the cosmic microwave background to the highly non-linear clustering hierarchy of galaxies, groups, clusters and voids we measure today; dark matter drives the clustering in the standard picture. However, Sanders (2001) argues heuristically, and Nusser (2002) and Knebe \& Gibson (2003) show, that stronger-than-Newtonian accelerations of the kind contemplated here do accelerate the rate of evolution to yield an approximation to the observed clustering hierarchy. As yet, it is unclear whether the results can be consistent with all data. The weakness of this argument is that we lack an appealing, generally covariant, alternative theory of gravity on which to base a self-consistent calculation without dark matter. It is therefore not possible to use structure formation arguments to distinguish dark matter models from alternative gravity models, because we do not yet know how the predictions in the two scenarios might differ.

While large-scale structure is a clear success for $\Lambda$ CDM, the properties of galaxies on smaller scales are more problematic. Sellwood \& Kosowsky (1999) give a now rather dated list of the difficulties confronting galaxy formation in the standard $\Lambda$ CDM model. Since other speakers at this meeting have expanded on these difficulties, I do not try to update this list here. Furthermore, it is generally argued that the shortcomings of the current model do not necessarily imply its failure, but may instead reflect the fact that the predictions are simply too crude. It is clearly desirable to continue to refine the predictions from the $\Lambda$ CDM model, but it seems unlikely that all problems will evaporate as calculations improve.

\section{Direct detection of WIMPs}

WIMPs are the most popular dark matter candidate. Their interaction crosssection with normal baryonic matter, while extremely small, is expected to be non-zero. We may therefore be able to detect them directly and a number of experiments are underway. Aside from the puzzling seasonal variation in their detection rate reported by the DAMA collaboration (Bernabei et al. 2003) all other experiments have so far failed to show a positive signal (e.g. Akerib et al. 2003; Sanglard et al. 2003; etc.), and some almost completely exclude a particle that could have been responsible for the DAMA result.

The absence of a positive detection is of no great significance at the current sensitivity levels. Most experiments barely reach the sensitivity required to 
detect even the most optimistic models for the properties of WIMPs (e.g. Cline 2003). Sensitivity is being improved all the time, but there is a long way to go before non-detections would begin to rule out interesting WIMP candidates.

It is expected that WIMPs and their anti-particles should be present in almost equal numbers. Annihilation radiation will be emitted at an enhanced rate from high-density regions, such as a dark matter cusp in the center of a galaxy (Bertone et al. 2001). Mayer-Hasselwander et al. (1998) report an EGRET detection of an unidentified gamma ray source in the Galactic center, but it is now believed not to be coincident with the Galactic center direction and is too concentrated to arise from annihilation radiation (Hooper \& Dingus 2002). The non-detection by EGRET does not tell us much about WIMPs in the Milky Way halo, since the densest part of the predicted cusp could easily have been erased by normal astrophysical processes (e.g. Merritt et al. 2002). The increased sensitivity of the future GLAST satellite may be sufficient to detect the expected diffuse gamma radiation (e.g. Wai 2002; but see also Evans et al. 2003). [Boehm et al. (2003) speculate that the INTEGRAL detection of $511 \mathrm{keV}$ radiation from the Galactic Center could be annihilation radiation from a low-mass dark matter particle.] A convincing detection of diffuse annihilation radiation would be conclusive evidence for the existence of dark matter in the form of WIMPs.

\section{Acoustic oscillations in the CMB}

Temperature fluctuations in the cosmic microwave background are believed to arise from acoustic oscillations seeded by primordial density fluctuations. Analysis of the power spectrum yields much information about the nature of the universe (Jungman et al. 1996). The first peak arises from the largest scale density variations that have just had time to collapse since they entered the horizon. The second peak, at higher $l$, occurs for smaller scale waves that entered the horizon at an earlier time and have had time to rebound to the maximum rarefaction. The third peak is on the scale of those waves that have achieved a second collapse, etc.

Since baryonic oscillations are damped (Silk 1968), the heights of the successive peaks should decrease monotonically in the absence of dark matter. Density variations in the dark matter, however, do not oscillate because there is no pressure term - the material interacts with neither the photons nor the baryons. The self-gravity of the photon-baryon plasma oscillating in the potential variations caused by the dominant dark matter causes the odd-numbered peaks (from collapses) to be higher relative to the even ones (from rarefactions), which is a generic signal of dark-matter driven oscillations (Hu \& Sugiyama 1996, Hu \& White 1996). Thus, we need to examine whether the third peak is higher than the second.

Unfortunately, the height of the third peak is not yet strongly constrained by the data. The best fit model (Spergel et al. 2003) places it at about the same height as the second peak, but the error bars on the first year WMAP data are quite large at this scale. The unconventional analysis by Odman (2003) suggests that other experiments may have systematic errors, since she suggests that the relative height of the third peak depends on the frequency of the observation, 
which should not be the case. The relative heights of the second and third peaks will not be determined until we have better data - perhaps soon to come from WMAP. A third peak clearly higher than the second would provide stronger, less model-dependent, evidence for dark matter than is currently indicated by the first year WMAP data (Spergel et al. 2003; see also McGaugh 2003).

\section{Light as a predictor of mass}

There is no doubt that Newtonian mechanics requires mass-to-light ratios that increase with increasing scale (e.g. Bahcall et al. 1995). Modified gravity theories, which are proposed in order to account for this fact, actually require much more: a unique relationship between the baryonic mass and the dynamical mass. This stringent requirement leads to a number of specific predictions that makes them much more easily falsified than is the dark matter hypothesis.

The relationship between the observed baryonic mass and the inferred Newtonian dynamical mass can, in principle, depend on spatial scale, or acceleration (as in MOND), or some other property, but the relation must be universal. Variations in the baryonic/dark mass ratio in similar objects could arise in dark matter models, but cannot be tolerated in modified gravity models. Two wellestablished examples where this holds are the Tully-Fisher relation for galaxies and the absence of bias in the galaxy distribution; the 2dF Galaxy Redshift Survey team (Verde et al. 2002) conclude that "optically selected galaxies do indeed trace the underlying mass distribution." Estimates of bias on smaller scales (e.g. Pen et al. 2003) are still subject to significant uncertainties.

Furthermore, modified gravity theories require the gravitational potential well to reflect the shape and orientation of the luminous matter. Dark matter halos, on the other hand, can have much more general shapes and may also be misaligned with the orientation of the luminous matter. These predictions are confronted by available data in this section.

\subsection{Detailed rotation curve shapes}

Kalnajs's (1983) dramatic demonstration that the inner parts of galaxy rotation curves can be predicted from the light distribution by a simple mass-traces-light model has been amply confirmed in many subsequent investigations (Kent 1986; Buchhorn 1992; Broeils \& Courteau 1997; Palunas \& Williams 2000; Sancisi, these proceedings). However, such models based on Newtonian dynamics generally fail at larger radii, where the predicted rotation curve falls below that observed.

The outstanding triumph of MOND is that it has yielded a formula that is able to predict the entire detailed rotation curve of a disk galaxy from the light profile. This achievement goes considerably further than its original motivation and works best for the galaxies for which the best data are available, as first shown for a sample of 10 galaxies by Begeman, Broeils \& Sanders (1996). Sanders \& McGaugh (2002) increase the sample to $\sim 75$ well-studied disk galaxies.

MOND rotation curve fits have only the mass-to-light ratio, $\Upsilon$, for the stellar component(s) as a free parameter. In some cases, the distance to the galaxy might require adjustment because the actual acceleration within the galaxy, 
$V^{2} / R$, is distance-dependent, while the characteristic acceleration $a_{0}$ that enters the fitting formula is not. Generally the fits are quite successful for the standard distance to the galaxy, but adjustments to improve the fits can be as large as a factor 2 in a few cases. A distance required for a good MOND fit could, in principle, be excluded by independent distance indicators, and the few cases where this could potentially invalidate MOND are discussed in Bottema et al. (2002).

Milgrom \& Sanders (2003) demonstrate that the declining rotation curves in elliptical galaxies recently announced by Romanowsky et al. (2003) are also in accord with MOND predictions. Gerhard et al. (2001) argue that mass discrepancies begin to appear in elliptical galaxies at much stronger accelerations, $\sim 10 a_{0}$, although their conclusion is also strongly challenged by Milgrom \& Sanders (2003). If it can be convincingly demonstrated that the value of $a_{0}$ is not universal, the success of MOND for spiral galaxies could no longer be interpreted as a manifestation of an unknown universal physical law.

It should be emphasized that conventional dark matter fits have three free parameters: $\Upsilon$, and two halo parameters, such as the core radius, $r_{0}$, and $V_{\text {flat }}$ for the pseudo-isothermal halo, or the concentration, $c$, and $V_{200}$ for an NFW halo. The success of MOND fits, with essentially only a single parameter, therefore cannot be dismissed lightly. If dark matter halos are, in fact, present in these galaxies, then the impressive success of the MOND fitting formula implies an intimate connection between the dark and luminous matter that presents a daunting fine-tuning problem for dark matter models.

Kaplinghat \& Turner (2002) attempt to show that a characteristic acceleration of the MOND value emerges "naturally" from the CDM model, but their claim is weak. They show only that the acceleration within a galaxy at which dark matter begins to dominate is of the order of MOND's $a_{0}$. Since their analysis uses the Gaussian initial fluctuation spectrum, they inevitably predict a spread in $a_{0}$ that is not required and they fail utterly to account for the spectacular accuracy of the MOND fits to the detailed rotation curve shapes.

\subsection{Dark lenses?}

The case for dark matter would be essentially made if we were able to discover objects with extremely high, almost infinite, M/L. The only way such objects might be discovered is through gravitational lensing. Schneider (this meeting) remarks that there are no known examples of strong lensing by a dark object, but a few such claims have been made from weak lensing.

Probably the strongest claimed detection of a dark cluster is by Erben et al. (2000), who find a "robust" lensing signal suggesting a previously unidentified dark mass concentration of perhaps $10^{14} \mathrm{M}_{\odot}$. There is no visible galaxy cluster and little X-ray emission from the position of this apparent mass concentration. However, it is hard to imagine how a real dark cluster-mass object could have avoided collecting enough hot gas to be X-ray bright. It is possible that the lensing signal is spurious and arises from intrinsic alignments among the background galaxies.

Weak lensing surveys, e.g. by Tyson's group (Jarvis et al. 2003), should reveal more such cases if they are common, but none has shown up so far. In fact, the lensing mass does not have to be completely dark to rule out alternative 
gravity theories; large variations in $\mathrm{M} / \mathrm{L}$ between similar lensing-mass clusters would be sufficient.

\section{Halo shapes}

Simulations of large-scale structure formation have revealed that the dark matter halos of galaxies and clusters are expected to be triaxial. The detailed distribution of halo shapes reported by the different groups varies slightly (see reviews by Springel and by Jing, these proceedings), but most authors find many nearly prolate shapes. It is also known (Dubinski 1994) that baryonic infall makes the halo more nearly axisymmetric.

A naive prediction from alternative gravity theories might be that the potential is flattened near a flattened light distribution and becomes more nearly spherical further away, since the higher order moments of the potential drop off more quickly. Aguirre (these proceedings) points out that this simple-minded expectation is not necessarily true, especially in MOND.

The meager data on the shapes of dark matter halos are reviewed by Sackett and by Arnaboldi (these proceedings). I continue to be impressed by the absence of a clear detection of a strongly non-axisymmetric extended HI disk that can be attributed to a triaxial dark matter halo. Bekki \& Freeman (2002) speculate that spirals in the outer disk of NGC 2915 arise from a rotating triaxial halo, but other interpretations are possible (e.g. Masset \& Bureau 2003). The startlingly round outer ring of IC 2002 (Franx et al. 1994) remains an isolated case. The lack of other cases could, perhaps, be a selection bias if gas in a non-axisymmetric halo is driven inwards by shocks until it reaches a closely axisymmetric potential.

\subsection{Misalignments}

Kochanek (2002) could find no evidence for misalignments between the luminous matter and the lensing mass distribution in a number of strong lens systems, and concluded that "Mass traces light." Perhaps yet more impressive is the claim by Hoekstra et al. (2003) of a detectable flattening of the dark matter halos in a statistical analysis of weak lensing data from stacking a large number of galaxies. If halos were mostly prolate, and the density axes at larger radii were twisted at random angles from those in the inner parts, the flattening found by Hoekstra et al. (2003) should not be detectable. (See Aguirre, these proceedings, for further discussion of their paper.)

Buote et al. (2002), on the other hand, argue that alternative gravity theories can be excluded by their claim of misalignment between the flattened x-ray halo of NGC 720 and the optical image. It is noteworthy that their estimate of the shape and orientation of the halo changed greatly from their earlier estimates based on ROSAT data to this recent analysis of Chandra data, due mainly to the elimination of point sources. The lumpy nature of the x-ray isophotes in even their cleaned Chandra image may indicate further contamination by fainter point sources, or it may indicate that the halo gas is subject to non-gravitational forces, or has has yet to settle. While this one case is not sufficiently compelling to rule out alternative gravity models, their research program is one of the few with this goal. 


\subsection{Substructure}

Dalal \& Kochanek (2002) argue that the flux ratio anomalies observed in a significant number of quadruple image lens systems indicate the presence of substructure in the dark matter halo. Convincing evidence for the mini-halos predicted in CDM models would constitute decisive support for dark matter. Mao (these proceedings) reviews the evidence, finding a number of puzzles, but concludes that mini-halos are the most likely explanation for flux anomalies, while Schechter (these proceedings) argues that alternative explanations are hard to rule out.

\section{Galaxy mergers}

Binney \& Toomre asked whether modified gravity theories could be consistent with galaxy mergers; specifically, whether galaxies that fall together could merge if there are no DM halos to take up the orbital energy and angular momentum? Binney \& Tremaine (1987) argue that a pair of similar, spherical galaxies approaching each other will dissipate their orbital energy and angular momentum and merge within a Hubble time for some limited range of specific orbital energies and angular momenta. In a modified gravity picture, the two galaxies would accelerate towards each other more strongly, and would therefore acquire much greater kinetic energy and angular momentum for their masses, making a merger less likely. The effect will scarcely be compensated by increased orbit-tointernal exchanges during the interaction, because forces during the encounter are closer to the Newtonian regime.

Mergers can occur even without dark halos, for sufficiently slow passages, as demonstrated for Newtonian dynamics by Toomre \& Toomre (1972). Modified gravity is unlikely to change this conclusion, but will make such encounters rarer. The question then becomes a quantitative one of whether the merger rate will be sufficiently suppressed by modified gravity to be inconsistent with that observed. A clear answer to this question requires a detailed calculation that has yet to be performed, and will depend on the nature of the modification. Two further points are worth making: First, Toomre's (1977) idea that elliptical galaxies are products of mergers seems to be holding up, but most were probably in place already at moderately high redshift (e.g. van Dokkum \& Ellis 2003), perhaps before the time that departures from Newtonian dynamics become significant (Sanders 2001). Second, Hickson compact galaxy groups seem to undergo rapid mergers (Barnes 1989) in conventional dark matter models, making the presentday frequency of these groups difficult to understand - but see also White (1990); modified gravity theories should ease this difficulty.

Studies of individual cases are always revealing. Dubinski et al. (1999) argue that tidal tails require rather low density dark matter halos in conventional dynamics. Comparable studies with modified gravity have not been reported, but would seem to be warranted. In particular, it would be very interesting to test whether simulations of merging systems with dark matter can account for the dramatically more concentrated stellar component in elliptical galaxies reported by Romanowsky et al. (2003). 


\section{Conclusions}

This very brief survey of the available data has turned up no really decisive evidence either in favour of dark matter or that could rule out alternative gravity theories. To form a scorecard, I regard the strongest evidence in favour of dark matter as the CMB power spectrum and the explanation of flux ratio anomalies in quadruple lenses in terms of halo substructure. On the other hand, new gravitational physics is favoured by the extraordinarily clear relationship between the light and mass distributions in galaxies, and the lensing evidence (Kochanek 2002, Hoekstra et al. 2003) that the mass distribution seems to be aligned with the light - Buote et al. (2002) notwithstanding. The evidence from halo shapes is too fragmentary to put in either column.

It needs to be stressed that the success of MOND fits to rotation curves and the alignment of lensing mass with the visible mass distribution create very serious fine-tuning problems if mass discrepancies are indeed caused by dark matter halos.

As always, more data will help; it is especially desirable to pursue $\mathrm{M} / \mathrm{L}$ variations, misalignments, substructure, etc., since alternative gravity ideas can be more easily falsified by such evidence than can dark matter models. Decisive evidence for dark matter may come soon from a firm measurement of the relative heights of the 2nd and 3rd peaks in the CMB, possibly even from WMAP, or from a detection of annihilation radiation by the future GLAST mission. In the present absence of decisive evidence on either side, the peculiarities highlighted here seem significant enough to encourage further attempts to develop alternative gravity theories from which testable predictions could be made.

Acknowledgments. I thank Arthur Kosowsky and Moti Milgrom for comments on the manuscript. This work was supported by grants from NASA (NAG 5-10110) and from NSF (AST-0098282).

\section{References}

Aguirre, A., Schaye, J. \& Quataert, E. 2001, ApJ, 561, 550

Akerib, D. S., et al. 2003, hep-ex/0306001

Arkani-Hamed, N., Dimopoulos, S., Dvali, G. \& Gabadadze, G. 2002, hepth/0209227

Bahcall, N. A., Lubin, L. M. \& Dorman, V. 1995, ApJ, 447, L81

Bahcall, N., Ostriker, J. P., Perlmutter, S. \& Steinhardt, P. J. 1999, Science, 284,1481

Barnes, J. E. 1989, Nature, 338, 123

Begeman, K. G., Broeils, A. H. \& Sanders, R. H. 1991, MNRAS, 249, 523

Bekenstein, J. D. \& Milgrom, M. 1984, ApJ, 286, 7

Bekki, K. \& Freeman, K. C. 2002, ApJ, 574, L21

Bernabei, R., et al. 2003, astro-ph/0311046

Bertone, G., Sigl, G. \& Silk, J. 2001, MNRAS, 326, 799

Binney, J. \& Tremaine, S. 1987, Galactic Dynamics (Princeton: Princeton University Press) 
Boehm, C., Hooper, D., Silk, J. \& Casse, M. 2003, astro-ph/0309686

Bottema, R., Pestaña, J. L. G., Rothberg, B. \& Sanders, R. H. 2002, A\&A, 393, 453

Broeils, A. H. \& Courteau, S. 1997, in Dark and Visible Matter in Galaxies, eds. M. Persic \& P. Salucci, ASP Conference Series 117, (San Francisco: Astronomical Society of the Pacific) p. 74

Buchhorn, M. 1992, PhD thesis, Australian National University

Buote, D. A., Jeltema, T. E., Canizares, C. R. \& Garmire, G. P. 2002, ApJ, 577,183

Carroll, S. M., Duvvuri, D., Trodden, M. \& Turner, M. S. 2003, astro-ph/0306438

Cline, D. B. 2003, astro-ph/0310439

Dalal, N. \& Kochanek, C. S. 2002, ApJ, 572, 25

Dubinski, J. 1994, ApJ, 431, 617

Dubinski, J., Mihos, J. C. \& Hernquist, L. 1999, ApJ, 526, 607

Erben, Th., van Waerbeke, L., Mellier, Y., Schneider, P., Cuillandre, J.-C., Castander, J. J. \& Dantel-Fort, M. 2000, A\&A, 355, 23

Evans, N. W., Ferrer, F. \& Sarkar, S. 2003, astro-ph/0311145

Franx, M., van Gorkom, J. H. \& de Zeeuw, T. 1994, ApJ, 436, 642

Gerhard, O., Kronawitter, A., Saglia, R. P. \& Bender, R. 2001, AJ, 121, 1963

Hinshaw, G., et al. 2003, ApJS, 148, 135

Hoekstra, H., Yee, H. K. C. \& Gladders, M. D. 2003, astro-ph/0306515

Hooper, D. \& Dingus, B. 2002, astro-ph/0210617

Hu, W. \& Sugiyama, N. 1996, ApJ, 471, 542

Hu, W. \& White, M. 1996, ApJ, 471, 30

Jarvis, M., Bernstein, G. M., Fischer, P., Smith, D., Jain, B. \& Tyson, J. A. 2003, AJ, 125, 1014

Jungman, G., Kamionkowski, M., Kosowsky, A. \& Spergel, D. N. 1996, Phys. Rev. D, 54, 1332

Kalnajs, A. J. 1983, in Internal Kinematics and Dynamics of Galaxies, IAU Symp. 100, ed. E. Athanassoula (Dordrecht: Reidel) p 87

Kaplinghat, M. \& Turner, M. 2002, ApJ, 569, L19

Kent, S. M. 1986, AJ, 91, 1301

Khoury, J., Steinhardt, P. J. \& Turok, N. 2003, hep-th/0307132

Knebe, A. \& Gibson, B. K. 2003, astro-ph/0303222

Kochanek, C. S. 2002, in The shapes of galaxies and their dark halos, ed. P. Natarajan (Singapore: World Scientific) p. 62

Kolb, E. W. \& Turner, M. S. 1990, The Early Universe (Reading: AddisonWesley)

Masset, F. S. \& Bureau, M. 2003, ApJ, 586, 152

Mayer-Hasselwander, H. et al. 1998, A\&A, 335, 161

McGaugh, S. S. 2003, astro-ph/0312570

Merritt, D., Milosavljevic, M., Verde, L. \& Jimenez, R. 2002, Phys. Rev. Lett, 88,191301 
Milgrom, M. 1983, ApJ, 270, 365

Milgrom, M. \& Sanders, R. H. 2003, ApJ, 599, L25

Nusser, A. 2002, MNRAS, 331, 909

Ödman, C. J. 2003, New Astron.Rev., 47, 741 (astro-ph/0305254)

Ostriker, J. P. \& Steinhardt, P. J. 2003, Science, 300, 1909

Padmanabhan, T. \& Choudhury, T. R. 2002, Phys. Rev. D, 66, 081301

Palunas, P. \& Williams, T. B. 2000, AJ, 120, 2884

Pen, U.-L., Lu, T., van Waerbeke, L. \& Mellier, Y. 2003, MNRAS, 346, 994

Romanowsky, A. J., et al. 2003, Science, 301, 1696

Sanders, R. H. 2001, ApJ, 560, 1

Sanders, R. H. 2003, MNRAS, 342, 901

Sanders, R. H. \& McGaugh, S. S. 2002, ARA\&A, 40, 263

Sanglard, V. et al. 2003, astro-ph/0306233

Sellwood, J. A. \& Kosowsky, A. 2001, in Gas and Galaxy Evolution, eds. J. E. Hibbard, M. P. Rupen \& J. H. van Gorkom, ASP Conference Series 240, (San Francisco: Astronomical Society of the Pacific) p. 311

Silk, J. 1968, ApJ, 151, 459

Spergel, D. N., et al. 2003, ApJS, 148, 175

Toomre, A. 1977, in The Evolution of Galaxies and Stellar Populations, ed.

B. M. Tinsley \& R. B. Larson (New Haven: Yale University Observatory), p 420

Toomre, A. \& Toomre, J. 1972, ApJ, 178, 623

van Dokkum, P. G. \& Ellis, R. S. 2003, ApJ, 592, L53

Verde, L. et al. 2002, MNRAS, 335, 432

Wai, L. 2002, astro-ph/0209121

Wang, L., Caldwell, R. R., Ostriker, J. P. \& Steinhardt, P. J. 2000, ApJ, 530, 17

Weinberg, S. 2000, astro-ph/0005265

White, S. D. M. 1990, in Dynamics \& Interactions of Galaxies, ed. R. Wielen (Heidelberg: Springer-Verlag), p. 380 